\title{
Colibrì: taking the pulse of black holes and neutron stars
}

Heyl, Jeremy, Caiazzo, Ilaria, Gallagher, Sarah, Hoffman, Kelsey, Safi-Harb, Samar

Jeremy Heyl, Ilaria Caiazzo, Sarah Gallagher, Kelsey Hoffman, Samar SafiHarb, "Colibrì: taking the pulse of black holes and neutron stars," Proc. SPIE 11444, Space Telescopes and Instrumentation 2020: Ultraviolet to Gamma Ray, 114442A (13 December 2020); doi: 10.1117/12.2562625

SPIE. Event: SPIE Astronomical Telescopes + Instrumentation, 2020, Online Only 


\title{
The Colibrì High-Resolution X-ray Telescope
}

\author{
Jeremy Heyl $^{\mathrm{a}}$, Ilaria Caiazzo ${ }^{\mathrm{b}}$, Sarah Gallagher ${ }^{\mathrm{c}}$, Kelsey Hoffman ${ }^{\mathrm{d}}$, Samar Safi-Harb ${ }^{\mathrm{e}}$, and the \\ Colibri collaboration ${ }^{\mathrm{f}}$ \\ a University of British Columbia, Vancouver, BC, Canada \\ ${ }^{\mathrm{b}}$ California Inst. of Technology, Pasadena, CA, USA \\ ${ }^{\mathrm{c}}$ Western University, London, ON Canada \\ 'Bishop's University, Sherbrooke, QC, Canada \\ ${ }^{\text {e}}$ University of Manitoba, Winnipeg, MB, Canada \\ fhttps://www.colibri-telescope.ca
}

\begin{abstract}
We propose a high-time-resolution, high-spectral-resolution X-ray telescope that uses transition-edge sensors (TES) as detectors and collector optics to direct the X-rays onto the focal plane, providing a large effective area in a small satellite. The key science driver of the instrument is to study neutron stars and accreting black holes. The proposed instrument is built upon two technologies that are already at high TRL: TES X-ray detectors and collector optics.
\end{abstract}

\section{KEY SCIENCE GOALS}

In the past 50 years, we have been able to observe what could be defined as the most amazing laboratories in the Universe: black holes and neutron stars. These objects, often called compact objects, uniquely present an environment to test the laws of physics at their extremes: the density in a neutron star reaches values several times higher than nuclear density, magnetic fields are billions of times higher than the Sun's, and gravity around black holes is so strong as to trap light itself. In this short period of time, we have learned a lot about compact objects, but their best kept secrets are still a mystery to us.

The X-ray emission of compact objects presents a rich phenomenology that can lead us to a better understanding of their nature and to address more general physics questions. The key scientific questions for Colibri can be divided in three main areas:

\section{Accretion physics in the strong gravity regions close to compact objects}

- What happens within the innermost region of the accretion disc around black holes and neutron stars?

- What is the structure of the X-ray emitting region of the accretion disc?

- What powers the corona? What drives X-ray weak and strong sources?

\section{Feedback mechanisms on all scales}

- What drives outflows in AGN? What are the properties (density, velocity, geometry, kinetic luminosity) of outflows?

- How do supernovae energize and enrich the environment with the elements essential for life? How do massive stars (that eventually explode in supernovae) lose mass and interact with their environment and what role do their magnetic fields play?

- Colibri is well-suited to addressing another science objective found within the Cosmic Origins topical team, but that falls into the feedback category, which is to characterize the highly ionized material of the hot intergalactic medium.

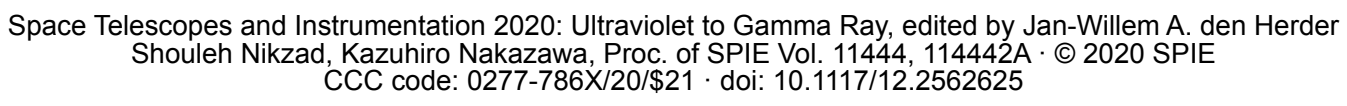

Proc. of SPIE Vol. 11444 114442A-1 


\section{Physics of dense matter and extreme magnetic fields}

- What is the equation of state for neutron star material, and how can we probe the interiors of neutron stars?

- What are the properties of material and light in strong magnetic fields?

Colibri will be able to investigate these questions to a new level, thanks to its unprecedented throughput and spectral and timing resolution in the $0.5-20 \mathrm{keV}$ range.

\subsection{Accretion physics in the strong gravity regions close to compact objects}

Variability in the X-ray emission from accreting neutron stars and black holes carries detailed information on the physics of accretion. Accretion disks and jets are ubiquitous in astrophysics; they are found around newborn stars, during planetary formation, in active galactic nuclei (AGNs), in which they play a key role in shaping the evolution of galaxies. However, the mechanisms for angular momentum transport in the disk and for jet formation close to the central object are poorly understood. As for any type of physics, studying accretion at its most extreme actualisation, the inner accretion disk close to compact objects, provides the best opportunity for breakthroughs in the understanding of the phenomenon as a whole. For example, the high magnetic field expected to be present in the disk of accreting stellar mass black holes, and even more so around accreting neutron stars, could make it easier to highlight the role of magnetic fields in generating the viscosity needed for accretion to occur. Or the role of the black hole spin in powering winds and jets can be better understood once we measure the spins of many black holes.

Additionally, the X-ray emission of accreting compact objects provides a window into strong gravity. One of the most subtle consequences of General Relativity is the "no-hair" theorem, for which black holes can be fully characterized by their mass, angular momentum and charge. Since we expect no charge on astrophysical black holes, the spacetime that surrounds a black hole can be nearly exactly described by the Kerr metric. The only way to test this theorem is to probe the spacetime very close to the hole. Fortunately, the X-ray emission of accreting black holes carries information about the inner region of the accretion disk, within a few gravitational radii $\left(R_{g}=G M / c^{2}\right)$ from the hole, encoded in the fast variability of its spectrum.

The emission from the accretion flow very close to accreting compact objects presents two possible highprecision diagnostics of their spacetime and accretion processes: reverberation mapping and quasi-periodic oscillations (QPOs).

Reverberation mapping In black-hole X-ray binaries and AGNs, accretion to the central black hole takes place via a geometrically thin, optically thick accretion disk, which emits thermally in the soft X-rays for black hole binaries and in the optical and UV bands for AGNs $[2,3]$. The photons emitted by the disk are thought to be Compton up-scattered in an optically thin corona, which produces a power-law spectrum in the hard $\mathrm{X}$-rays $[4,5]$. Some of the up-scattered photons in the corona are reflected back into the line of sight by the disk. The reflected spectrum presents particular features, that include an iron $\mathrm{K} \alpha$ fluorescence line at $6.4 \mathrm{keV}$ and a reflection hump that peaks at $\sim 30 \mathrm{keV}[6,7]$. Gravitational redshifts from the black hole and relativistic motion of the orbiting plasma in the inner disk distort the spectrum, providing insight on the dynamics of the accretion disk [8]. The coronal emission shows rapid aperiodic variability, on timescales of milliseconds for stellar mass black holes and of minutes for AGNs. Neutron stars also accrete via a disk, but in addition, the material accreted onto the surface causes repeating thermonuclear reactions that are observed as bright bursts of X-ray emission, with timescales that go from $100 \mathrm{~ms}$ to several hours [9-16]. Similar to the coronal emission for black holes, this X-ray-burst emission can be reflected by the accretion disk, and indeed reflection spectra have been observed [17-20]. In both cases, variability of the illuminating signal provides a way to map the inner regions of the accretion disk, since fluctuations in the continuum emission are reflected in the reverberation spectrum with a light-crossing time delay[21]. Time delays can be of the order of a few hundreds of microseconds for Galactic binaries, and are much longer for AGN (scaling as $\propto M$ ). Since different parts of the accretion disk will be illuminated at subsequent times, photons of different energy will present different time delays, reflecting the 


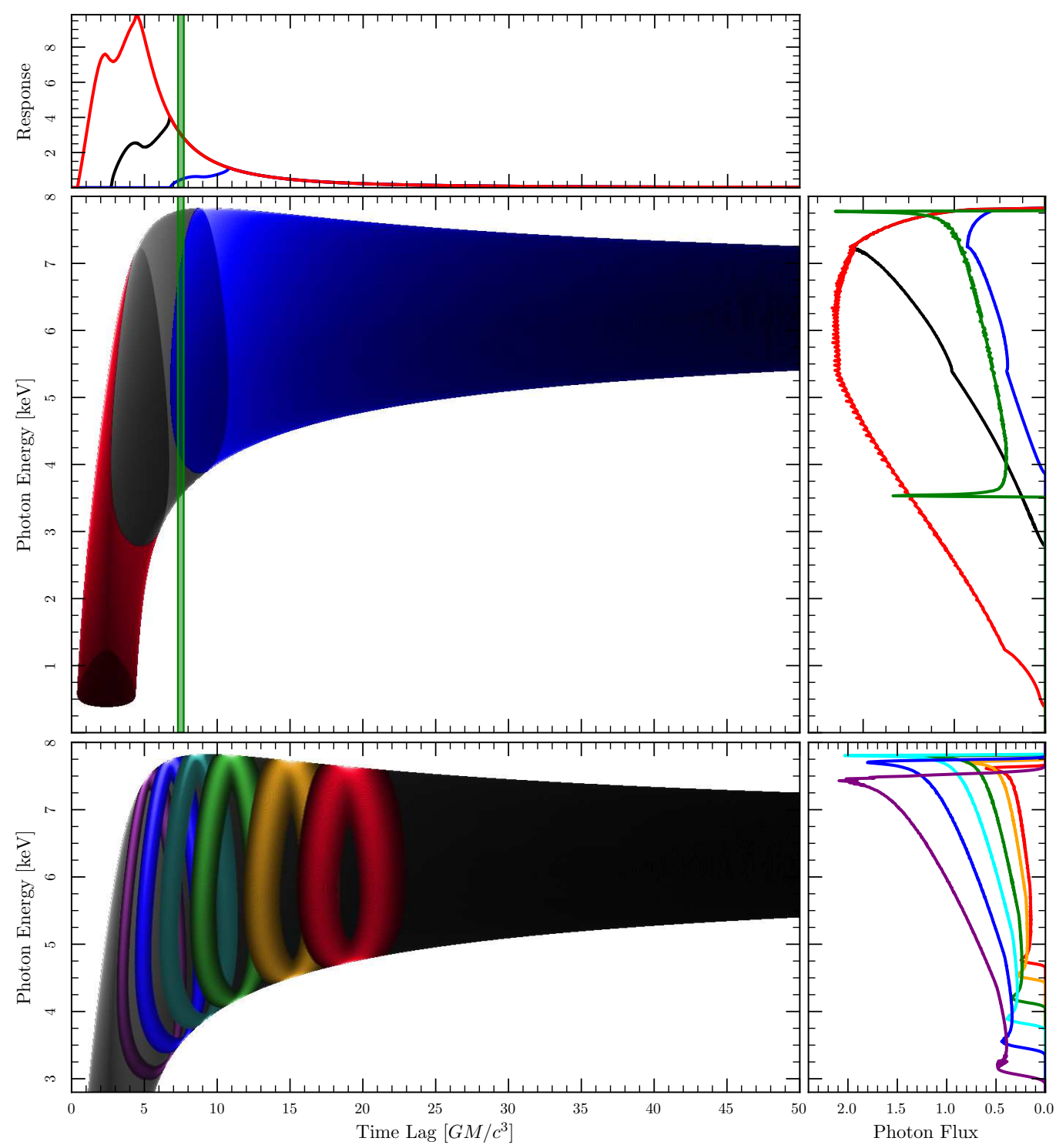

Figure 1: Energy- and time-dependent reflected emission resulting from a $\delta$-function (the iron line) in the driving continuum for a black hole with spin parameter $a / M=0.998$. The corona is modelled as a point source along the spin axis at $z=2 G M / c^{2}$ (lamppost), and the observer is located at an inclination of $86^{\circ}$. See also [1]. Upper part: Central panel. Reverberation response spectrum for the accretion disk ending at the ISCO (red), $4 G M / c^{2}$ (black) and $8 G M / c^{2}$ (blue). Upper panel. Energy integrated flux. Photons of different energies, coming from different radii on the disk, also arrive at different times. Right panel. Time integrated spectrum. The caustics form at the edge of the response function as can be seen in the reverberation spectrum measured a particular time lag (green). Lower part: Central panel. The colored regions depict the reverberation signal from parts of the disk where a QPO of $Q \sim 10$ and the nodal precession frequency of 1,2, 5, 10, 20 and $40 \mathrm{~Hz}$ would exist for a $10 \mathrm{M}_{\odot}$ black hole, assuming the RPM model. Right panel. The caustics can be seen in the reflection spectrum measured for a particular QPO. 
characteristic Doppler shift of the reflection region. Such reverberation lags have been detected in several AGNs with XMM-Newton and NuSTAR [22, 23], and very recently in an X-ray binary with NICER [24].

Observations so far are limited by either the low sensitivity or low timing resolution of current X-ray telescopes, while the combination of high energy- and high time-resolution of Colibri will allow for the detection of these lags in the reverberation spectra. High-resolution spectral fitting of the X-ray emission, especially of the Fe-line profile, provides information on the strong-field gravity effects on the orbiting plasma and its dynamics, from which radii can be inferred in units of the gravitational radius, as well as the spin of the black hole and the inclination angle of the system. The possibility of performing reverberation mapping, which yields distances in absolute units given by the light travel time, simultaneously to spectral fitting would therefore provide a test of the Kerr metric itself, as well as a measurement of the mass of the compact object $[25,26]$. Although the total spectral features of reverberation are relativistically broadened in general, high energy and timing resolution combined would allow slicing the emission in both the spectral and time domain, which could reveal sharp features, broadened not by the bulk motion of the disk material but by thermal and turbulent motion within the disk with $v / c \sim 10^{-3}$ or smaller as shown by the green curves in Figure 1.

Quasi periodic oscillations QPOs are nearly periodic fluctuations commonly observed in the X-ray light curve from the inner regions of accreting compact objects [27-30]. The first to be discovered were the low frequency QPOs (LFQPOs), with frequencies $\sim 0.1-30 \mathrm{~Hz}$. RXTE revealed higher frequency features: high frequency QPOs (HFQPOs) in the range $\sim 40-450 \mathrm{~Hz}$ from black hole systems [31], and $\mathrm{kHz}$ QPOs in the range $\sim 300-1200 \mathrm{~Hz}$ in neutron star systems [29, 32]. The origin of QPOs is still debated, but their frequencies are commensurate with those of orbital and epicyclic motions in the Kerr metric close to the compact object, and thus constraining the QPO mechanism would provide a new way to measure properties of the inner accretion flow and the effects of strong gravity.

Current models for LFQPOs find their origin either in some instability in the accretion flow, or in a geometric oscillation [33-40] such as, most notably, Lense-Thirring precession [34, 35, 37-39]. This is a nodal precession of orbits inclined to the equatorial plane caused by a spinning compact object dragging the surrounding spacetime around with it (the frame-dragging effect). The origin of HFQPOs and $\mathrm{kHz}$ QPOs is more obscure, with the proposed models including Doppler modulation of orbiting hotspots in the inner disk, oscillation modes of a pressure-supported torus, nonlinear resonances, gravity and pressure modes in the accretion disk [41, 42, and references therein] and, for the case of neutron stars, beating with the neutron star spin frequency [43].

It is clear that new and better observations are needed to understand these phenomena and ultimately exploit them as diagnostics. LFQPOs are normally detected with high significance using current instruments, thanks to their high amplitudes. This enables studies of the QPO phase dependence of the spectral shape (i.e. QPO tomography). Recently, Ingram et al. [44] detected the predicted quasi-periodic modulation of the iron line centroid energy using XMM-Newton and NuSTAR data from the black hole XRB H1743-322, and performed a tomographic mapping of the disk emission [45]. It was a challenging task, as it required very long exposure times, and a relatively dim source was chosen to avoid photon pile-up in the XMM-Newton detectors. Colibri will revolutionize such studies by providing vastly better spectral resolution and dramatically higher count rates, particularly considering that instruments such as XMM-Newton (and ATHENA in the future) are limited by photon pile-up and therefore cannot be used to observe the brightest sources. Furthermore, the unprecedented count rates will, for the first time, enable similar tomographic analyses with $\mathrm{HF}$ and $\mathrm{kHz}$ QPOs, providing a qualitatively new way of testing models. HFQPOs are much fainter than LF, and this explain the scarcity of current detections. The high sensitivity of Colibri will enable the detection of HFQPOs in more systems and it will test the presence of the even weaker signals predicted by some of the current theoretical models. The observed HFQPOs with RXTE show a similar set of frequencies from all the sources. This may be a selection effect from the RXTE band, or it could mean that HFQPOs are excited only at specific frequencies. A higher sensitivity and timing resolution could bring to the detection of additional signals or to a null detection at higher frequencies, allowing to discern between the proposed models. Perhaps most important in the study of QPOs is detecting large number of photons and high time resolution; nevertheless, combining this with high energy resolution could allow a new type of diagnostic on the origin of QPOs: if the modulation is coming from a 

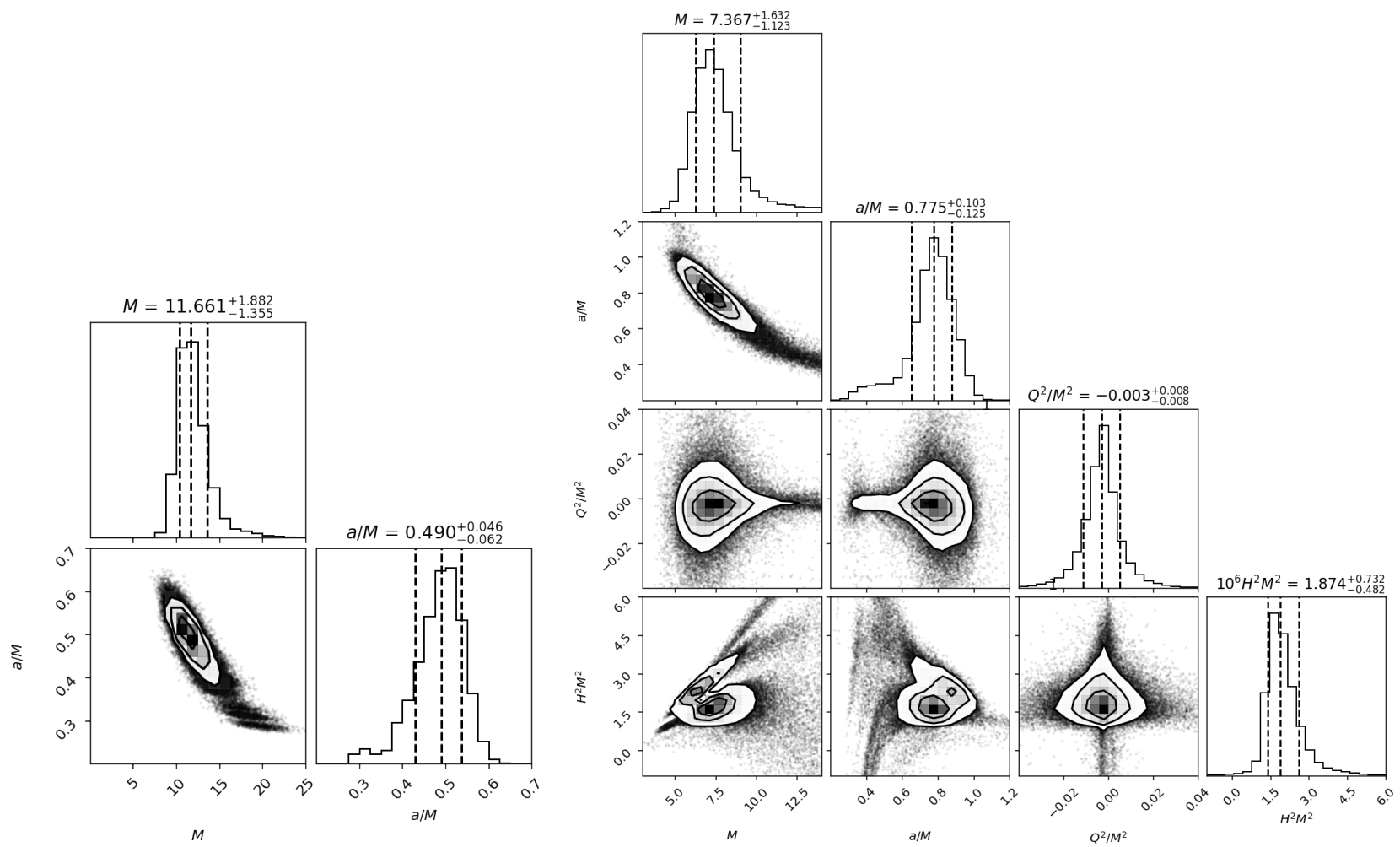

Figure 2: Left: Constraints on the mass and spin of the black hole in XTE J1550-564 obtained using the frequencies in observed in [46] assuming that the black hole lacks charge and vacuum energy as expected for astrophysical black holes. Right: Constraints on the mass and spin of the black hole as well as the additional parameters that we expect to be zero from GR for astrophysical black holes ( $Q$ and $H)$.

small area in the disk, slicing the emission in energy would provide information on where the perturbation is happening.

As an example of the power of QPOs as diagnostic, Fig. 2 shows the results of a simulation for a HFQPO on a $10 \mathrm{M}_{\odot} \mathrm{BH}$ rotating at $95 \%$ of the critical spin performed for Colibri where the relativistic precession model (RPM) for HFQPOs was assumed. In the RPM, four signals are expected in the power spectrum: LenseThirring precession, radial epicyclic motion, periastron precession and orbital motion, and the relation between the frequencies is uniquely determined by the Kerr metric. A drift in the frequencies is observed, correlated to a variability in the flux, that is interpreted in the RPM as a response to a change in the inner radius of the disk. Observations of the four signals and of how they drift, not only would shed light on the origin of the phenomenon, it would constrain the mass and spin of the hole with great accuracy, as shown in Fig. 2. Furthermore, since mass and spin are the only parameters important in describing the spacetime around a black hole in the Kerr metric, these observations could provide constraints on deviations from it. In Fig. 2 a simple test is shown: adding a charge or a cosmological constant, both expected to be negligible in astrophysical black holes, changes the metric from Kerr; finding a value different from zero would hint to a deviation from the Kerr metric and from GR.

If we turn our focus to accreting neutron stars, the highest-frequency quasi-periodic oscillations (QPOs) observed in accreting neutron star systems can provide unique constraints on the neutron stars themselves [47] if the oscillation can be associated with motion near the inner edge of the accretion disk. The current record is $4 \mathrm{U} 0614+09$, which has a QPO with three-sigma lower limit on its frequency of $1267 \mathrm{~Hz}$, yielding a constraint on the mass of this object of less than $2.1 \mathrm{M}_{\odot}$ [48]. High-resolution spectroscopy of the QPO itself can bring the power of these constraints forward to obtain stellar mass measurements and probe the spacetime around the neutron star as well (perhaps measuring the moment of inertia). The objects that have been found to exhibit these high frequency QPOs are typically brighter than 0.1 Crab (this is in part a selection effect). They are 
sufficiently bright that a focusing instrument like the $X$-IFU on ATHENA [49] would have significant difficulty doing spectroscopy due to photon pile-up, but a TES experiment with collecting optics could provide exciting measurements of neutron star masses and moments of inertia, as well as a basic test of the models for the QPOs.

Testing General Relativity The detections by the LIGO experiment over the past four years of gravitational radiation from inspiraling black holes represent the most spectacular verification of the unification of gravity with special relativity. The existence of gravitational waves is the most basic feature of a relativistic theory of gravity. It is direct proof that the disturbances in spacetime itself travel at the speed of light. The discovery, on 17 August 2017, of gravitational waves from merging neutron stars and a nearly coincident gamma-ray burst constrained the speed of gravitational waves to be within one part in $10^{15}$ of the speed of light. Furthermore, gravitational waves are a window into gravity at its most dynamic: during the final moments of the inspiral detected in September 2015, the energy released by the two black holes in the form of gravitational radiation exceeded the energy released in all forms by the rest of the Universe in that moment.

General relativity is one among many possible relativistic theories of gravity that are consistent with the current constraints in the weak-field regime and with the observed LIGO events. In this context, Colibri can play a crucial role in probing gravity in the strong field near black holes and neutron stars and in putting constraints on the different theories. The timing capabilities of Colibri will yield complementary probes of strong gravity. One of the most subtle consequences of general relativity is the "no-hair" theorem, which states black holes can be fully characterized by their mass, angular momentum and charge. In an astrophysical context, this means that the spacetime outside of black holes is nearly exactly described by the Kerr metric. By measuring multiple QPOs from multiple black holes, Colibri will not only measure the spin and mass of black holes, but provide crucial constraints on the departure of the spacetime from the Kerr metric and a verification of the "no-hair" theorem. Through spectroscopic studies of accretion disks around black holes, e.g. by looking at the reverberation spectrum or by mapping the iron line, it will be possible, with Colibri, to probe the fine scale structure of the spacetime around black holes, both within the equatorial plane and above it.

Although gravitational waves provide a window to strong gravity, the wavelength of these waves is necessarily comparable to the size of the black holes; therefore, without extremely high-signal-to-noise, the study of gravitational waves cannot provide a detailed probe of the spacetime surrounding black holes, and electromagnetic signals provide a crucial complementary window into strong gravity.

\subsection{Feedback mechanisms on all scales}

Most galaxies are known to host a supermassive black hole at their centre; additionally, a tight correlation is observed between the mass of the host galaxy and the mass of the supermassive black hole. This observational correlation requires a mechanism that connects the accretion growth of the black hole to the star formation growth of the galaxy. Understanding this feedback mechanism is crucial to the study of how the galaxies and the black holes at their centre evolve.

Actively accreting black holes are among the brightest extra-galactic X-ray sources in the sky. As the Xrays travel through space, the spectrum is imprinted with the signatures of all of the material along the line of sight. This includes the material in the immediate vicinity of the black hole, including energetic winds that are launched from the system. Further away from the hole, X-ray photons travel out of the host-galaxy of the black hole and through the reservoir of gas and dust that is the intergalactic medium. In particular, as the X-rays travel through the hot filamentary web of the warm-hot intergalactic medium (or WHIM), some photons can be absorbed by the highly ionized metals contained in the gas. From these absorption lines in the spectra, we can measure how much gas is contained in these otherwise dark reservoirs.

A different feedback mechanism is important at a smaller scale: as stars die, their ultimate explosions, supernovae, energize and enrich with elements the gas in star-forming regions. The study of supernova remnants with the high spectral resolution and high throughput of Colibri will enable a better understanding of the composition of the outflows and of the different types of supernovae. 

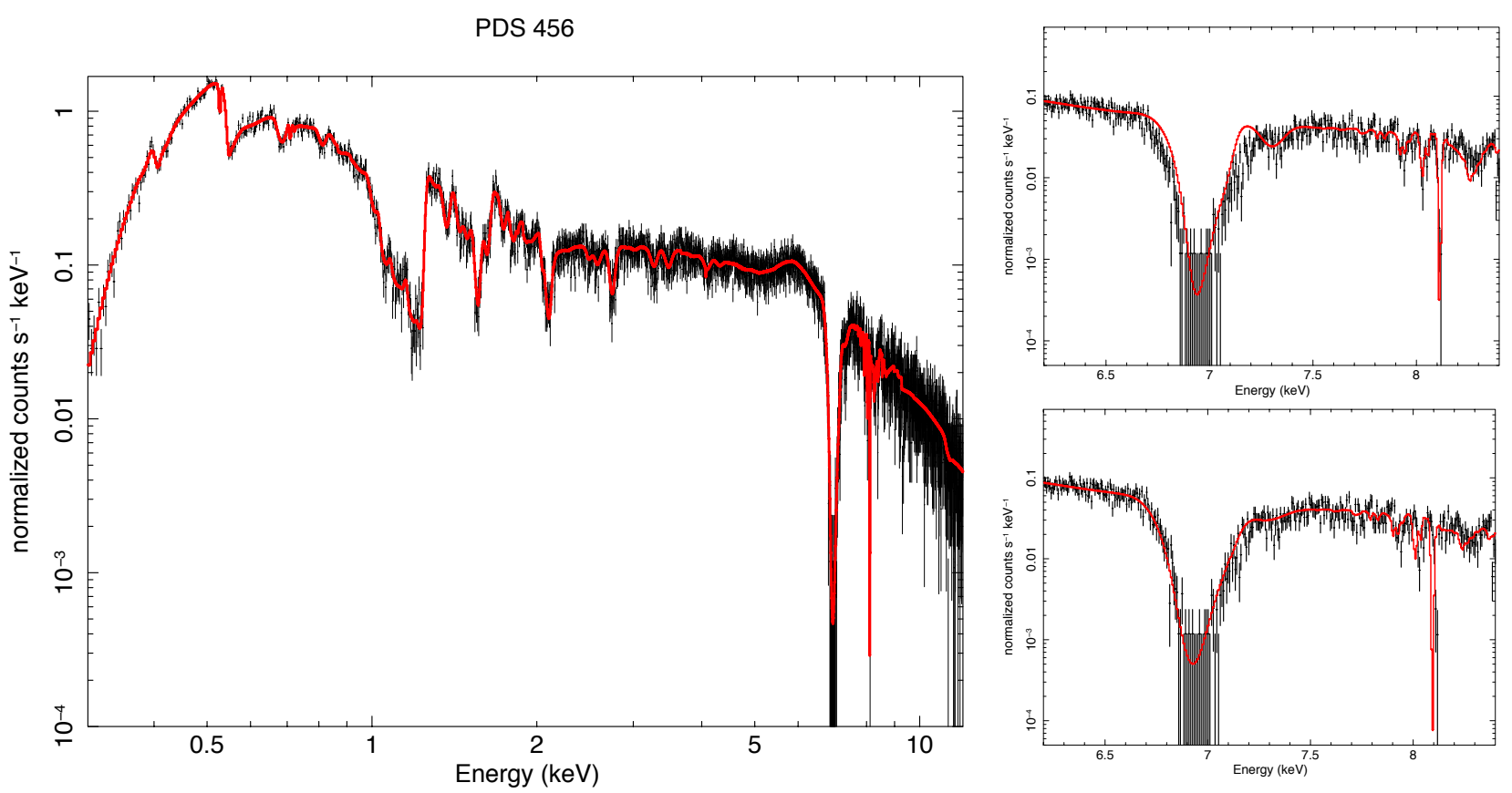

Figure 3: Left: Simulations of a 100 ks observation of absorption by the ultra-fast outflow from the quasar PDS 456 at about $7 \mathrm{keV}$ with Colibri. Upper right: The observations are sensitive to the turbulent velocity broadening of the outflow $(3000 \mathrm{~km} / \mathrm{s}$ and $5000 \mathrm{~km} / \mathrm{s})$. Lower right: The observations also probes the mean velocity; the velocities of the model and data differ by one percent.

Black hole winds The most significant feedback mechanism is seen when active galaxies are in "radio mode" and launch collimated jets of material across the intracluster medium. In "quasar mode", diffuse winds are released from the accretion disc region into the host galaxy. While the quantity ejected in quasar mode may be more modest than in radio mode, it can still have a profound effect on star formation and the distribution of elements in the host galaxy. These more common outflows appear in nearly half of all AGNs, exhibit velocities of hundreds up to $\sim 1000 \mathrm{~km} \mathrm{~s}^{-1}$, and are seen in optical, UV, and X-ray spectra.

With the large effective area at high energies $(\sim 5-10 \mathrm{keV})$ provided by XMM-Newton, came the discovery of absorption features above $7 \mathrm{keV}$ [e.g. 50, 51]. These features were attributed to highly ionized Fe XXV and XXVI that are outflowing from the inner regions. The blueshifts indicate the material is moving at mildly relativistic velocities of about $10^{4}-10^{5} \mathrm{~km} \mathrm{~s}^{-1}$ and have been coined ultra-fast outflows (UFOs). If confirmed, UFOs would correspond to the hottest, densest, and fastest gas expelled from the black hole system and dispelled on the host galaxy.

Determining the nature of UFOs may be the key to understanding how galaxies grow and evolve. However, the modest spectral resolution of current instruments means little is known about the wind. The high spectral resolution and throughput of Colibri will reveal the composition of the wind and the launching mechanism.

Supernova Remnants Supernovae energize and enrich the environment with the elements essential for life. As such, they are the main sources for the chemical enrichment of the Universe and our own galaxy. Supernova remnants (SNR) serve as laboratories to probe these elements through X-ray spectroscopic studies and comparison to nucleosynthesis models developed by nuclear astrophysicists. However, the details of these important processes and how elements are created and dispersed into the interstellar medium remain unknown to date, largely due to the lack of high-resolution spectroscopy needed to resolve spectral lines and constrain the abundances of the elements. In particular, the following questions remain to be answered: what is the mechanism behind the type Ia thermonuclear explosions of white dwarfs (accretion from a non-degenerate star, merger of 


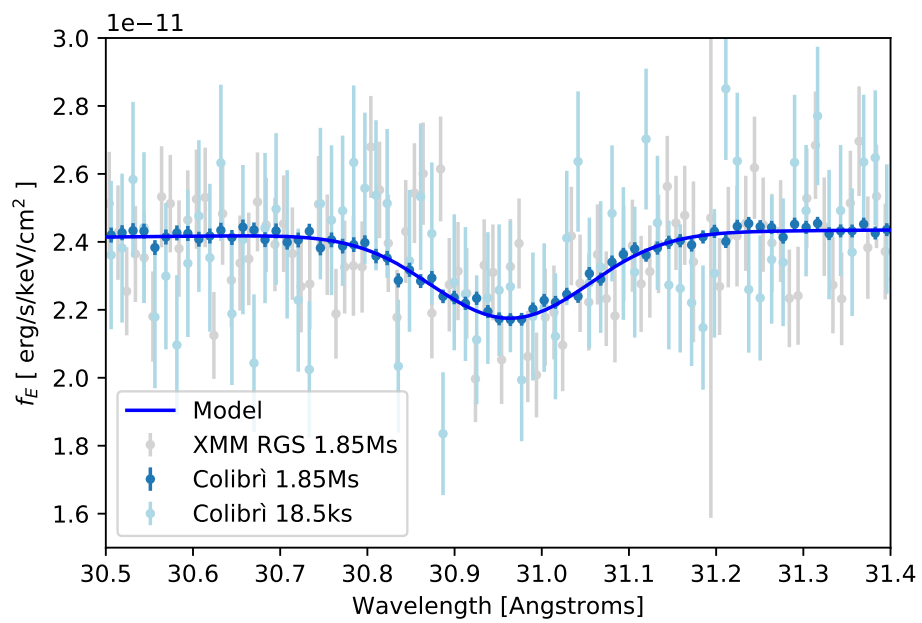

Figure 4: A simulation of the observations of the WHIM along the line of sight to the blazar 1ES 1553+113 with $X M M R G S$ [as in 52] and Colibri. Here we focus of the $\alpha$-line of helium-like oxygen from an absorber at $z=0.4433$.

two white dwarfs, or both)? Can we probe the heavy, rare and odd-Z elements (like $\mathrm{Mn}$ and $\mathrm{Cr}$ ) beyond the most abundant even-Z species detected with CCDs? Is the observed diversity of neutron stars, born in corecollapse supernovae, linked to that of their supernova progenitors? The answer lies in future high-throughput, high-energy resolution spectroscopy, as hinted by Hitomi's glimpse of a handful of SNRs and the Perseus Cluster. Colibri can serve as a future instrument for high-throughput, high-resolution spectroscopy of a sample of diffuse objects that do not require spatially resolved studies.

Finding the missing baryons Ultimately the feedback from supernovae and AGN not only affects the star formation of the host galaxy but also energises the surrounding gas and pollutes it with material from the galaxy itself. Bright quasars illuminate the gas along the line of sight, i.e. the gas between the galaxies in intergalactic space. With its high spectral resolution and high sensitivity, Colibri will enable detection and characterization of this material through absorption features.

Analysis of the density fluctuations in the cosmic microwave background, as well as observations of the high-redshift intergalactic medium (IGM) through Lyman- $\alpha$ absorption, provide an estimate of the fraction of baryonic matter in the Universe. Recent Planck results estimate this baryon fraction to be 4.86\% [53]. Of this fraction, about $10-20 \%$ is found in galaxies, groups and clusters, while the rest is expected to lie in the reservoirs of gas that make up the IGM. However, observations of the IGM at low redshift account only for about half of the remaining $80-90 \%$ of baryons. A big question then arises: where are the missing baryons in our current epoch?

Cosmological simulations suggest that the gas in the IGM at low redshift is distributed over a wide range of temperatures and densities, which are usually divided into two main components: a diffuse and photoionized phase, with temperatures of the order $T \sim 10^{3.0-4.5} \mathrm{~K}$ and electron densities of about $n_{e} \sim 10^{-8}-10^{-5} \mathrm{~cm}^{-3}$, and a condensed $\left(n_{e} \sim 10^{-6}-10^{-4} \mathrm{~cm}^{-3}\right)$ filamentary web that is heated by shocks to temperatures $T \sim 10^{5-7} \mathrm{~K}$ [54]. The diffuse phase can be traced by detecting Lyman- $\alpha$ absorption lines in the spectrum of background quasars and contains about $25 \%$ of the low-redshift baryons [55]. The condensed and hot phase is called the warm-hot intergalactic medium (or WHIM), and it is thought to contain the remaining $60 \%$ of the gas in the IGM [56].

Due to the large abundance of hydrogen in the collisionally ionized WHIM, it is possible to detect broad Lyman- $\alpha$ absorption from a small neutral fraction at temperatures up to $T \sim 10^{6} \mathrm{~K}$ [57, 58]. Other successful surveys of low-z WHIM at $T<10^{6} \mathrm{~K}$ were obtained by observations of high ionization resonance lines in the far 
or extreme ultraviolet (as for example Oxygen VI or Neon VIII lines) [55, 59, 60]. The two methods have some overlap, and the estimates of the fraction of baryons contained in the warm phase of the WHIM are of the order of $20-30 \%[52,56]$.

The remaining 30-40\% of elusive baryons may be hidden in the hotter portion of the WHIM, and searching for them requires detecting the metal absorption lines in the $\mathrm{X}$-rays from highly ionized $\mathrm{C}, \mathrm{O}$ or Ne. The throughput of current high-resolution spectrographs on Chandra or XMM-Newton is too low to obtain significant detections of these absorption lines in the spectra of bright background quasars in reasonable exposure times; however, several controversial detections have been claimed of absorption lines of helium-like Oxygen VII [61-63] or hydrogen-like Oxygen VIII [64, 65]. Recently, an extremely long observation (1.85 Ms) with XMM-Newton of the brightest X-ray blazar in the sky with $z>0.4$ (1ES 1553+113) has produced a $3-\sigma$ detection of two helium-like Oxygen VII absorption lines which are likely to originate in the IGM and not in the quasar's environment [52]. A similarly significant detection of a O VII line was obtained with a much shorter (470 ks) observation of the luminous quasar H $1821+643$, by blue-shifting and stacking the X-ray spectrum corresponding to the redshift of 17 absorption line systems in the quasar's sightline previously detected in the UV [66]. Fig.4 shows the sensitivity of Colibri to the weak absoption lines of the WHIM.

\subsection{Physics of dense matter and extreme magnetic fields}

The density reached in a neutron star's core, several times higher than nuclear density, is not reached anywhere else in the universe at cold temperatures, let alone in our terrestrial physics labs, and therefore neutron stars represent the only laboratory available to look for the equation of state for cold, dense matter. The holy grail of neutron star observations, the mass-radius relation, if measured for several neutron stars, could put stringent constraints on the nuclear equation of state, and thus on the detailed physics and composition of matter at high density [67]. Mass measurements of massive neutron stars exclude a number of equations of state that predict a relatively "soft" dependence of pressure on density, as the nuclear pressure must be high enough for massive neutron stars to be stable. Although a number of masses of neutron stars have been measured with high precision, especially for compact binaries, radius measurements are much harder to achieve with the precision of less than a kilometre required to put stringent constraints on the equation of state.

The advent of moderately high-resolution X-ray spectroscopy with Chandra and XMM-Newton promised to usher in a new age in the study of neutron stars: we thought we would study neutron stars like stars, with resolved absorption spectra revealing their surface chemical composition and physical conditions (e.g. surface gravity, pressure, temperature). Nature, however, did not cooperate in this endeavor, as observations of neutron stars have not revealed verified atomic absorption lines from their surfaces yet. Colibri will allow us to study neutron stars in much greater detail by achieving high-energy resolution with much larger collecting areas, to uncover even weak spectral features over a wide range of photon energies.

High energy-resolution, high-throughput spectroscopy of the neutron star surface Due to the high surface gravity of neutron stars, the elements in neutron star atmospheres stratify within 30 seconds, leading to a photosphere made from only the lightest element present, typically hydrogen. In most neutron stars, some amount of accretion (from a companion star, the interstellar medium, or fallback from the supernova) will have occurred, so featureless (in the X-ray) hydrogen atmospheres are generally expected [e.g. 68].

However, a variety of elements may be present in the photospheres of neutron stars, if the neutron stars are actively accreting. On rapidly spinning neutron stars, spectral lines will be spread out by the Doppler shift; therefore, detecting and measuring the energy and width of the spectral lines from a rotating neutron star would directly provide an estimate of the neutron star radius, if the spin period is known [69]. In general, rapid rotation may make the lines too broad to be detected. However, the narrow cores of these broad lines may be deep enough to be clearly detected with appropriate throughput and spectral resolution [70]. Also, some X-ray binaries have relatively low spin (e.g. Terzan $5 \mathrm{X}-2,[71])$, and/or very low inclination, either of which would narrow the lines sufficiently for possible detection [72].

During active accretion episodes, the surface of the neutron star is generally not visible, as photons from the stellar surface are Comptonized by the accreting material. Thermonuclear X-ray bursts represent an exception, as 

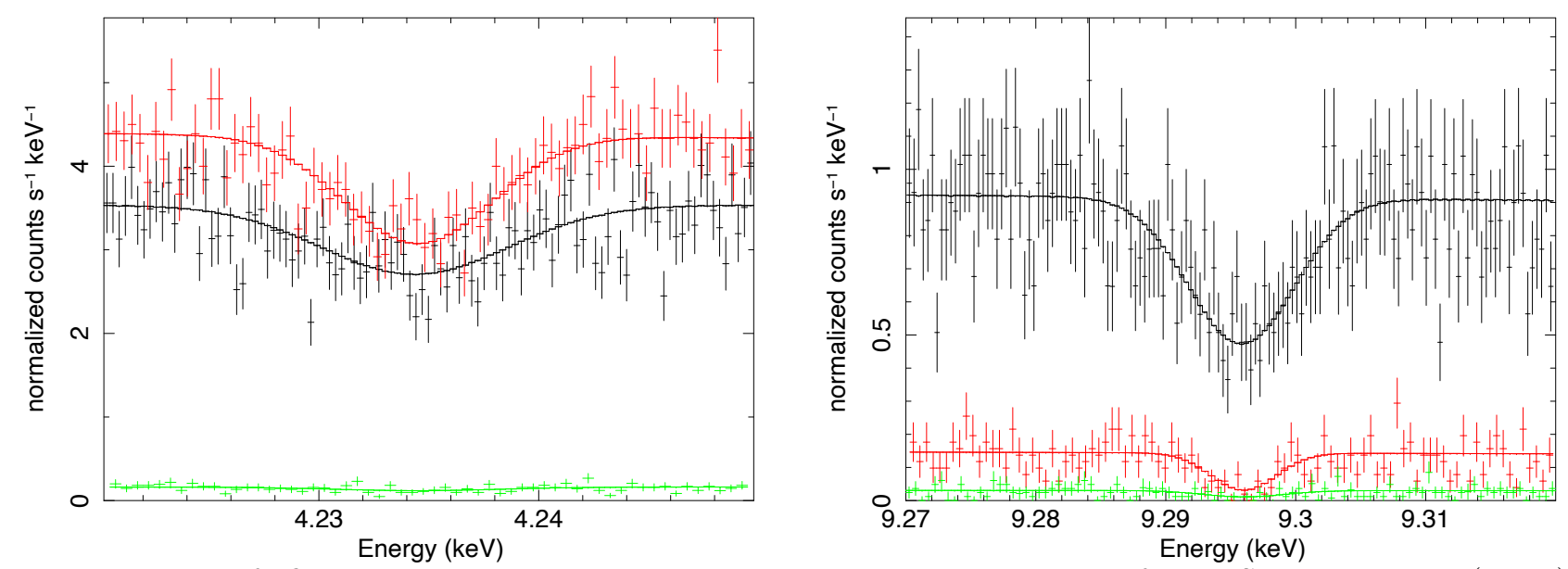

Figure 5: The left figure shows the line detected with Hitomi at $4.2345 \mathrm{keV}$ from PSR J1833-1034 (green) compared to simulations for ATHENA (red) and Colibri (black) for the same observing time. The figure on the right is the comparison of the Hitomi, ATHENA and Colibri detection of the line at $9.296 \mathrm{kev}$.

the reactions on the surface of the star dramatically increase the emission from the surface itself, so it dominates the X-ray emission for a few seconds to hours. Cottam et al. [73] identified absorption lines in the sum of XMM-Newton spectra over many Type-I X-ray bursts from EXO 0748-676, which they argued were redshifted Fe lines from the stellar surface. This particular source is now thought to be rotating rapidly [74], which makes it challenging to explain the relatively narrow spectral features that they found [70, 75].

However, several X-ray bursts since then have shown evidence for broader features, likely due to heavy nuclear burning products being mixed up to the photosphere in particularly energetic bursts [76]. These include observations of a likely edge around $7.5 \mathrm{keV}$ in HETE J1900.1-2455 by RXTE [77], and $8 \mathrm{keV}$ in GRS 1747-312 by $R X T E$ [78]. However, RXTE's spectral resolution was insufficient to clearly identify the spectral feature, while Colibri will have the spectral resolution to clearly resolve edge features such as these, and the effective area to spot them in short time periods $(\sim 1 \mathrm{~s})$, allowing robust determination of the surface redshift of these bursting neutron stars.

These particular observations are hard for planned instruments such as the X-IFU on ATHENA [49], because of photon pile-up, but straightforward for Colibri. The count rate during X-ray bursts will peak at about 1$10 \mathrm{kHz}$ (scaling from RXTE results [15]) for ATHENA and Colibri, which both plan to use TES X-ray detectors for spectroscopy. In the case of ATHENA's baseline design, if two photons arrive within $2.6 \mathrm{~ms}$ from each other on the same pixel, the energy of the second photon cannot be measured. The expected count rates for X-ray bursts dramatically exceed this limit, and therefore, without blocking filters to reduce the effective area to the level of XMM-Newton or deliberate defocusing, ATHENA cannot perform spectroscopy during X-ray bursts. On the other hand, because a mission with collecting optics such as Colibri builds effective area by having many collectors operating in parallel, with each collector focusing X-rays on several elements of a TES array, its nominal configuration can achieve high-resolution spectroscopy to count rates well beyond $100 \mathrm{kHz}$.

Low-mass X-ray binaries during quiescent periods between outbursts also exhibit surface features. If no accretion is occurring, then the photosphere will contain only the lightest element (typically $\mathrm{H}$ or $\mathrm{He}$ ), and no lines will be present. However, if accretion onto the neutron star exceeds $\dot{M} \sim 10^{-13} \mathrm{M}_{\odot} /$ year (corresponding to $L_{X} \sim 10^{33} \mathrm{erg} / \mathrm{s}$ ), it is likely that metals will substantially populate the photosphere. Evidence for metal features in the photosphere of the quiescent LMXB Aquila X-1 was produced by Rutlege et al. [79] from one (of several) Chandra observations. However, Chandra's spectral resolution and low-energy calibration left the nature of this feature in some doubt. Colibri will permit clear identification of these spectral features, likely within the context of a program to monitor the cooling of neutron star crusts after an outburst [80], when occasional burps of accretion are often observed. 
High-energy-resolution, high-time-resolution spectroscopy of the neutron star surface Many neutron stars are also rapidly rotating. Observing phase-resolved spectral features adds the requirement of hightime-resolution to high-throughput. Here, Colibri builds upon the heritage of RXTE and NICER. In particular, we plan to have an effective area larger than NICER, while using similar concentrators so that the count-rates will be similar to or larger than RXTE but extend to softer X-rays. Furthermore, unlike RXTE and NICER, Colibri will have high-resolution spectroscopy along with high-time resolution. Rotation imparts a particular pattern in the observed X-rays as a function of energy and phase. In particular, if only a portion of the surface is emitting, hard X-rays will lead softer ones [81], and if the emission pattern is known or can be constrained from observations as it could be in Type-I X-ray burst oscillations [82], one can constrain the mass and radius of the neutron star $[83,84]$. The boost in effective area of Colibri relative to NICER will allow us to study fainter objects in shorter times and to derive constraints from ensembles of Type-I X-ray bursts with oscillations. The dramatic increase in energy resolution will probe and constrain the underlying emission models, reducing potential systematic errors in the determinations of mass and radius.

Recently, the Hitomi satellite opened a new window in high-resolution spectroscopic studies of neutron stars. During a calibration observation of the supernova remnant G21.5-0.9 hosting the rotation-powered pulsar PSR J1833-1034, the Soft X-ray Spectrometer $(S X S)$ led to the surprising discovery of two spectral features at $4.2345 \mathrm{keV}$ and $9.296 \mathrm{keV}$ [85]. We have simulated this observation for Hitomi, ATHENA and Colibri with the results presented in Fig. 5. The red and black points show the simulated results with ATHENA and Colibri. Colibri will also open the possibility of phase-resolved spectroscopy to verify that the feature indeed originates from the pulsar rather than the hosting supernova remnant. PSR J1833-1034 rotates with a period of $66 \mathrm{~ms}$, so the rotational velocity at the equator of the neutron star is about $1000 \mathrm{~km} / \mathrm{s}$, yielding a width of about $15 \mathrm{eV}$, just a factor of two larger than Hitomi's energy resolution. If the line indeed originates at the surface, it is somewhat puzzling that Hitomi discovered such a narrow feature.

Strong evidence for spectral lines has been found for several slowly rotating neutron stars with stronger magnetic fields. The X-ray dim isolated neutron star (XDINS) RX J1308.6+2127 exhibits a spectral feature at about $740 \mathrm{eV}$ with an equivalent width of about $15 \mathrm{eV}$ [86] over only a portion of its rotation. Unfortunately, the energy resolution of the Epic-pn instrument on XMM-Newton is insufficient to resolve the line. A comprehensive analysis of the available data for the XDINS with XMM-Newton yields upper limits on the equivalent width of narrow (unresolved lines) of $10-50 \mathrm{eV}$, depending on the source and the duration of the available observations. Colibri, thanks to its energy resolution of about $1 \mathrm{eV}$, with a similar effective area to XMM-Newton would yield constraints ten times stronger for similar observing times.

Magnetars are among the most magnetic compact objects in the Universe. Their high energy properties and spin parameters point to a super-strong magnetic field of the order of $10^{14}-10^{15} \mathrm{G}$. Spectroscopy provides a direct diagnostic of their total surface magnetic field strength. While their electron cyclotron features would fall in the $\mathrm{MeV}$ band, their proton cyclotron lines fall in the X-ray band. To date, we have evidence of sporadic detections of spectral features in several magnetars' X-ray spectra, in outburst or quiescence (e.g., a $5 \mathrm{keV}$ absorption line from SGR1806-20 [87]; $8.1 \mathrm{keV}$ absorption line from 1RXS J170849-4009104 [88]; $4 \mathrm{keV}$ and $8 \mathrm{keV}$ emission lines from $4 \mathrm{U} 0142+62[89]$ ). While the interpretation of these lines is still being debated, they have been mostly interpreted as proton cyclotron features from a magnetar-strength magnetic field, confirming in many cases the high magnetic field value inferred from spin-down measurements. More recently a variable absorption feature near $2 \mathrm{keV}$ was discovered in phase-resolved spectroscopy of the magnetar SGR 0418+5729 whose spin properties point to a much lower magnetic field $\left(6 \times 10^{12} \mathrm{G}\right)$ below the QED value, supporting high-order multipolar field components [90]. This line, when interpreted as a proton cyclotron feature, yields a magnetic field ranging from $2 \times 10^{14}-10^{15} \mathrm{G}$. This suggests that spectroscopy can directly probe the topology of the magnetic field, in ways that cannot be done with timing, which only infers the dipole field strength. Furthermore, the data depicted hint at a second feature on the opposite hemisphere of the star. Observing similar lines in more neutron stars and with the higher sensitivity of Colibri could reveal the structure of the magnetic field and how magnetars work.

While early theoretical predictions suggested relatively wide absorption lines [e.g. 91, 92] as observed in some of the magnetar burst spectra, vacuum polarization has been subsequently suggested to suppress the strength of the proton cyclotron resonances in strongly magnetized plasma [93, 94]. This could reduce the line equivalent 
width by nearly an order of magnitude. Colibri will open a new window for a higher sensitivity search for the proton or ion cyclotron features (or atomic lines from high $\mathrm{Z}$ elements) with weak (shallow or narrow) lines, and will be especially suited to studying bright burst spectra and monitoring the evolution of magnetars' spectra.

High-time-resolution, high-throughput spectroscopy of fast spinning neutron stars Among the zoo of neutron stars, there is an important class of millisecond-period rotation-powered X-ray pulsars that have been used by NICER to constrain the neutron star equation of state. These pulsars spin fast with frequencies ranging from $200-300 \mathrm{~Hz}$, and their pulsations have been detected in radio, X-ray, and in some cases gamma-rays. None of these pulsars are accreting material from a companion, and their X-ray pulsations are thought to arise from heating of the star's surface at the magnetic poles by the return currents that are the mechanism behind the radio and gamma-ray pulsations. The pulsations are remarkably stable, allowing instruments such as NICER to observe for short periods of time and then phase-connect the short observations into one long observation. These pulsars have been key science targets for the NICER mission, as they allow a measurement or constraints on the masses and radii of selected pulsars, as well as providing new information about their magnetic field geometries.

NICER is currently observing the X-ray pulsations of a handful of pulsars with the goal of measuring their masses and radii. The method of pulse-profile modelling [95-97] uses relativistic ray-tracing to predict how X-ray photons travel from the hot spot on the star to the telescope. Since the light originates from anisotropies on the surface, the resulting X-rays are pulsed. The pulse profile, a plot of intensity versus spin phase (that also depends on photon energy) has information about the spacetime geometry encoded in its shape. The strength of the gravitational field depends on the ratio of the mass to the radius (also known as the compactness ratio), which controls how curved the path of the light is near the neutron star. Large compactness ratios curve the paths of photons more strongly, making it possible to view larger fractions of the star, and reducing the pulsation amplitude. Meanwhile, large speeds introduce Doppler effects which introduce harmonics into the pulse profile. Combined with a physical model for the atmosphere, it is possible to infer the neutron star's mass and radius, and constrain the equation of state of dense matter.

Among this class of pulsars, two exhibit large masses (near 2.0M $\odot$; PSR J1614-2230 and PSR J0740+6620), as determined from independent radio observations. NICER has detected X-ray pulsations from both of these pulsars, however both are very faint so the pulse profiles have low signal-to-noise. These two pulsars are of high scientific interest for two reasons. First of all, with such high masses, they may be close to the maximum mass allowed by the neutron star equations of state. Determination of the radius of such a high mass neutron star has the potential to provide very useful constraints on the equation of state. Secondly, even with low statistics, observations of pulsations provide interesting limits on the neutron star's radius. Since a large mass to radius ratio will allow for a larger part of the star's surface to be seen, a very small star with high mass will tend to have a low pulse fraction. The detection of a significant pulse fraction will then give a strong lower limit on the neutron star's radius.

These two high-mass pulsars J1614 and J0740 are potentially excellent targets for Colibri. Observation of either or both pulsars will provide a significant improvement in the signal-to-noise and help strengthen constraints on the physics of dense matter. In particular, the rapidly rotating $(3.15 \mathrm{~ms})$ and massive $\left(1.97 \pm 0.04 \mathrm{M}_{\odot}\right)$ pulsar PSR J1614-2230 is an excellent target [98] for high-time-resolution spectroscopy with Colibri. Pulsations have been detected by NICER [99], however it is somewhat too faint for a long targeted NICER observation that would constrain the neutron star's radius from a detailed analysis of its pulse profile. Observing PSR J1614-2230 with greater effective area could yield powerful constraints on the radius of this neutron star as well as the emission mechanism to control systematics. Given that this is a faint source, pile-up is not an issue. Because the field of this star is weak and it has not recently accreted, we do not expect to see spectral features, so the high-energy resolution is not crucial.

In parallel, similar science can be achieved when tackling another class of X-ray pulsars in both the hard and soft X-ray bands. Accretion-powered millisecond-period X-ray pulsars (AMXPs) are neutron stars in LMXBs that exhibit X-ray pulsations when the binary system goes into outburst. The stable pulsation periods, generally a few milliseconds, are most easily explained by identifying the pulsation period with the neutron star's rotation. The rapid rotation implied by the AMXP pulsations is comparable to the fastest rotation-powered pulsars (described above), which is evidence that the millisecond rotation-powered pulsars evolved to their rapidly spinning state 
Table 1: Key Mission Parameters

\begin{tabular}{ll|ll}
\hline Baseline requirements & & Optics & \\
Energy range & $0.5-20 \mathrm{keV}$ & Focal length & $4.9 \mathrm{~m}$ \\
Spectral resolution & $2-5 \mathrm{eV}$ & Number of Arrays & 7 \\
Timing resolution & $250 \mathrm{~ns}$ & Foils per Array & 30 \\
Effective area & $3,000 \mathrm{~cm}^{2}$ & Inner Radius & $4 \mathrm{~cm}$ \\
Count rate & $>100 \mathrm{kHz}$ & Outer Radius & $24 \mathrm{~cm}$ \\
Orbit & & Geometry & Conical Wolter I \\
Altitude & Coating & Iridium \\
Inclination & $1,688 \mathrm{~km}$ & Field of View & 50 arcseconds \\
Period & $103.0^{\circ}$ & Angular Resolution & 6 arcseconds \\
Sun Synchronous & 120 minutes & Detectors & TES Bolometers \\
Payload & & Bath Temperature & $70 \mathrm{mK}$ \\
Size & $2.1 \mathrm{~m} \mathrm{x} \mathrm{7.0} \mathrm{m}$ & $T_{c}$ & $100 \mathrm{mK}$ \\
Mass & $2,000 \mathrm{~kg}($ fixed bench) & Array & $12 \times 12(50 \mu \mathrm{m} \mathrm{pitch})$ \\
Power & Lifetime & minimum 5 years \\
\hline
\end{tabular}

through the accretion of material from a companion during an earlier LMXB phase. More recently the discovery of the transitional pulsars that are seen to transform from an accreting phase to a rotation-powered radio pulsar stage and back provides further evidence that the AMXPs are the progenitors of the millisecond rotation-powered pulsars.

The underlying model for an AMXP is that the neutron star's magnetic field lines trap material from the accretion disk, funnelling it onto the neutron star's magnetic poles [100]. The accreted material creates a hot spot on the star's surface which radiates as a soft $(\sim 1 \mathrm{keV})$ blackbody. The blackbody photons are inverse Compton scattered to a harder spectrum by energetic electrons near the star's surface. This leads to X-ray pulsations in the soft and hard parts of the X-ray spectrum. Since the pulsed X-rays are emitted at (or very close to) the neutron star's surface, analysis of the pulse profiles could yield information about the neutron star's mass and radius, using the same methods employed for the rotation-powered pulsars described above.

\section{TECHNICAL OVERVIEW}

The key characteristics of Colibri are its high spectral resolution, high throughput and large effective area, and they can all be achieved by using already mature technologies: thin-foil nested X-ray mirrors and transition-edge sensors as single-photon X-ray bolometers. Please consult Tab. 1 for an overview of the mission parameters. An array of seven telescopes yields a total effective area slightly larger than the three XMM-Newton telescopes with a focal length of 4.9-metres (see Fig. 6). The reduced focal length is achieved by reducing the outer diameter of each telescope to $32 \mathrm{~cm}$ (compared to the $70 \mathrm{~cm}$ for XMM-Newton) and by optimizing the on-axis performance at the expense of performance off-axis. This results in a large effective area for high-resolution spectroscopy from $0.2 \mathrm{keV}$ to $20 \mathrm{keV}$ as shown in Fig. 7

Colibri offers a similar energy resolution to the gratings on Chandra and XMM-Newton and to the bolometers on Hitomi SXS, but with ten times the effective area of these missions. It gives a similar energy resolution to ATHENA with lower effective area below about $6 \mathrm{keV}$, but higher above $6 \mathrm{keV}$, because of the mirror-design choices.

The second key characteristic of Colibri is high throughput combined with high-time resolution. The optics are purposefully defocused so that the emission from a source on axis is spread over a large portion of a TES array. Furthermore, seven arrays operate in parallel. This dramatically reduces the likelihood of several photons landing on the same detector element within a short time, which would result in poor energy measurements, pile-up and lost photons, and allows to sustain count rates a factor of 1,000 larger than ATHENA. Furthermore, because the individual detector elements relax more quickly than those planned for ATHENA, and there are seven arrays operating in parallel, Colibri will over-perform ATHENA by more than a factor of ten in count rate even if ATHENA offers a defocused observing mode. Colibri's rapid sampling of the TES arrays will achieve 


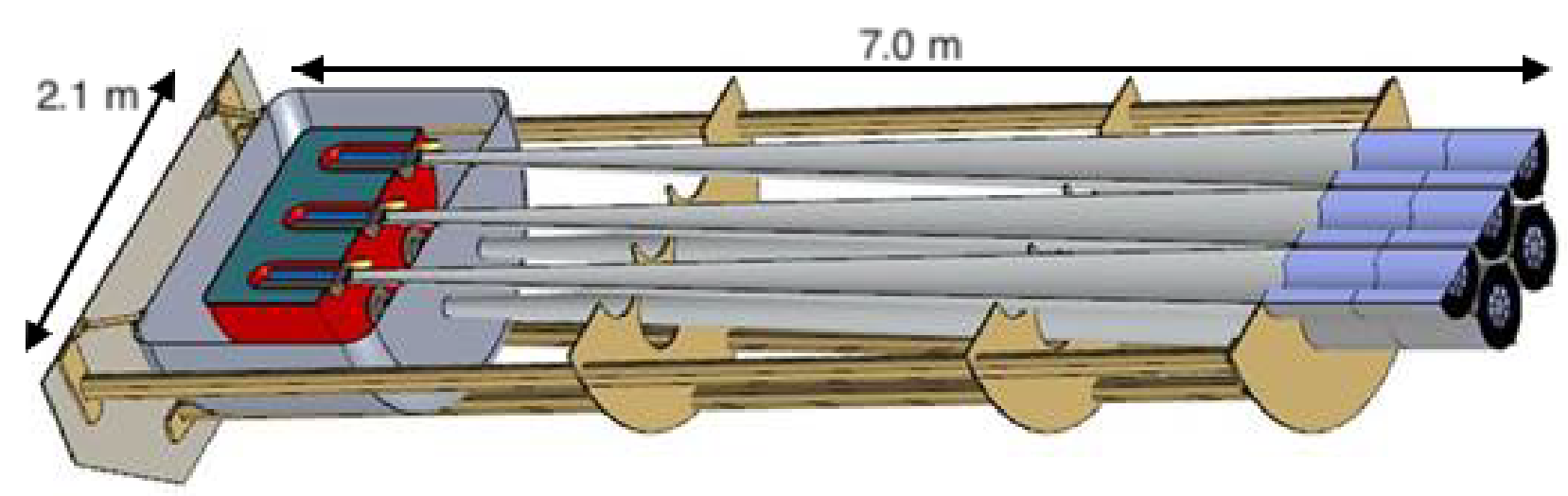

Figure 6: Cut-Away of Colibri to highlight the optical path, detectors and cooling concept.
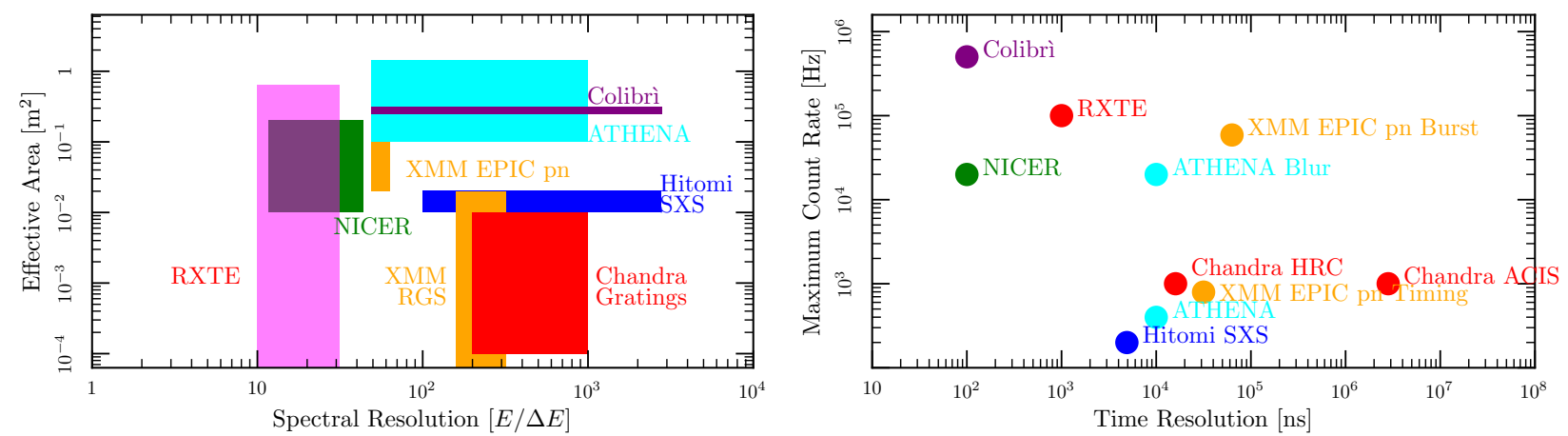

Figure 7: Left panel: X-ray Missions with X-Ray Spectroscopy Figures of Merit: Effective area and Spectral resolution at $1 \mathrm{keV}$ and $10 \mathrm{keV}$. Right panel: X-ray Missions with X-Ray timing figures of merit: time resolution and throughput

a time resolution similar to that of NICER and a factor of one-hundred finer than ATHENA. Fig. 7 depicts a summary of past, current and planned missions in the X-ray-timing space.

\section{TECHNOLOGY DRIVERS}

At its heart, Colibri relies on TES-enabled X-ray bolometers with small relaxation times and high sampling rates. These detectors, combined with non-focusing optics, yield high throughput, high spectral and timing resolution and high quantum efficiency over a wide range of photon energies. The performance that is required for Colibri's bolometers has already been achieved in the laboratory, and similar detectors are already in use at X-ray beamlines. Colibri could be the first mission to use these detectors in space. Although Colibri requires a cryogenic focal plane, the individual detector arrays and the accompanying superconducting electronics are much smaller than the focal planes of recent CMB experiments (e.g. Planck HFI at $100 \mathrm{mK}$ ) that have required similar operating temperatures; Hitomi's SXS used a similar technology as Colibri, and operated also at 50-100 mK. Furthermore the X-ray optics are well tested; telescopes with similar or better performances have been used on many missions.

The first key technology challenge is the on-board pulse processing that is required to achieve the energy and time resolution. Each of nearly two thousand TES bolometers must be sampled every 5 microseconds, for a total sampling rate of $400 \mathrm{MHz}$. We have developed a scheme of linear filters to achieve the required energy and time-resolution that will limit the required on-board computation to a manageable level. For example, Hitomi's SXS pulse processor was limited to count rates below $200 \mathrm{~Hz}$ by computational constraints. We plan to achieve counts of $100 \mathrm{~s}$ of $\mathrm{kHz}$ from sources with Crab-like fluxes.

The second key challenge is to bring the data back to the ground. During a day-long observation of a bright source, Colibri will amass about $50 \mathrm{~GB}$ of data (assuming just five bytes per photon), twenty times the mean rate 
for HST and one-fifth that of JWST. However, with Colibri in low-Earth orbit, the time each day to downlink data to a single ground station is dramatically diminished. We are exploring three options: a high-frequency (e.g. Ka-band as for JWST) downlink to a single ground station, downlink via satellites in high Earth orbit (as for Hubble) and an optical downlink to a telescope ground station. The final option potentially offers the most flexibility and room for growth but also is the most technically challenging.

The total power requirement for cooling the focal plane is about 1600 Watts. Although we do not yet have power estimates for other systems, cooling will play a major part of the power budget for the mission.

\section{ORGANIZATION, PARTNERSHIPS, AND CURRENT STATUS}

The Colibri collaboration consists of most of the Canadian high-energy astrophysics community teamed up with Canadian particle and condensed matter physicists as well as other scientists around the world. We recently completed an 18-month science concept study funded by the Canadian Space Agency, with Honeywell and MDA as industrial partners. During the study, we developed the optical design and cooling concepts, as well as a detailed plan for the payload and spacecraft bus. We have identified several additional potential partners: NASA (mirrors and TES detectors), NIST (TES detectors) and the Canadian Light Source (testing and calibration), NASA/ESA (launch).

\section{SCHEDULE}

The proposed Colibri mission has just completed the science concept study stage, in March 2020, which defined the mission success criteria as well as the mission requirements. In an optimistic timeline, the mission definition phase will continue for 4 years. During this time, science maturation studies and technology development studies will proceed in parallel to the mission definition in order to increase the science and technology readiness levels.

Over the subsequent 6 years, activities leading to launch will include defining the system requirements, a preliminary system design and a detailed system design, and then fabricating, integrating and testing the system. A data analysis pipeline will also be developed. This will lead to a mission launch in about 10 years, in the early 2030s. After the commissioning activities, the nominal science operations are estimated to occur over 5 years. Decommissioning activities are estimated to take 2 additional years, with the safe disposal of the Colibri space mission and final data archiving with documentation to ensure the usefulness of Colibri data long after the mission is completed.

\section{Acknowledgements}

The authors would like to acknowledge support from the Canadian Space Agency, the Natural Sciences and Engineering Research Council of Canada, the Canada Foundation for Innovation and Compute Canada.

\section{References}

[1] Mastroserio, G., Ingram, A., and van der Klis, M., "Multi-time-scale X-ray reverberation mapping of accreting black holes," MNRAS 475, 4027-4042 (Apr. 2018).

[2] Shakura, N. I. and Sunyaev, R. A., "Black holes in binary systems. Observational appearance.," A\&A 24, 337-355 (1973).

[3] Novikov, I. D. and Thorne, K. S., "Astrophysics of black holes.," in [Black Holes (Les Astres Occlus)], Dewitt, C. and Dewitt, B. S., eds., 343-450 (1973).

[4] Thorne, K. S. and Price, R. H., "Cygnus X-1 - an interpretation of the spectrum and its variability," ApJ 195, L101-L105 (Feb. 1975).

[5] Sunyaev, R. A. and Truemper, J., "Hard X-ray spectrum of CYG X-1," Nature 279, 506-508 (June 1979).

[6] Ross, R. R. and Fabian, A. C., "A comprehensive range of X-ray ionized-reflection models," MNRAS 358, 211-216 (Mar. 2005). 
[7] García, J., Dauser, T., Reynolds, C. S., Kallman, T. R., McClintock, J. E., Wilms, J., and Eikmann, W., "X-Ray Reflected Spectra from Accretion Disk Models. III. A Complete Grid of Ionized Reflection Calculations," ApJ 768, 146 (May 2013).

[8] Fabian, A. C., Rees, M. J., Stella, L., and White, N. E., "X-ray fluorescence from the inner disc in Cygnus X-1," MNRAS 238, 729-736 (May 1989).

[9] Belian, R. D., Conner, J. P., and Evans, W. D., "The discovery of X-ray bursts from a region in the constellation Norma," ApJ 206, L135-L138 (June 1976).

[10] Grindlay, J., Gursky, H., Schnopper, H., Parsignault, D. R., Heise, J., Brinkman, A. C., and Schrijver, J., "Discovery of intense X-ray bursts from the globular cluster NGC 6624," ApJ 205, L127-L130 (May 1976).

[11] Woosley, S. E. and Taam, R. E., "Gamma-ray bursts from thermonuclear explosions on neutron stars," Nature 263, 101-103 (Sept. 1976).

[12] Maraschi, L. and Cavaliere, A., "X-ray bursts of nuclear origin?," in [X-ray Binaries and Compact Objects], van der Hucht, K. A., ed., 127-128 (1977).

[13] Cornelisse, R. et al., "Six years of BeppoSAX Wide Field Cameras observations of nine galactic type I X-ray bursters," A\&A 405, 1033-1042 (July 2003).

[14] Cumming, A., Macbeth, J., in 't Zand, J. J. M., and Page, D., "Long Type I X-Ray Bursts and Neutron Star Interior Physics," ApJ 646, 429-451 (July 2006).

[15] Galloway, D. K., Muno, M. P., Hartman, J. M., Psaltis, D., and Chakrabarty, D., "Thermonuclear (Type I) X-Ray Bursts Observed by the Rossi X-Ray Timing Explorer," ApJS 179, 360-422 (Dec. 2008).

[16] Keek, L. and in't Zand, J. J. M., "On burning regimes and long duration X-ray bursts," in [Proceedings of the 7th INTEGRAL Workshop], 32 (2008).

[17] Ballantyne, D. R. and Strohmayer, T. E., "The Evolution of the Accretion Disk around 4U 1820-30 during a Superburst," ApJ 602, L105-L108 (Feb. 2004).

[18] Cackett, E. M., Miller, J. M., Ballantyne, D. R., Barret, D., Bhattacharyya, S., Boutelier, M., Miller, M. C., Strohmayer, T. E., and Wijnands, R., "Relativistic Lines and Reflection from the Inner Accretion Disks Around Neutron Stars," ApJ 720, 205-225 (Sep 2010).

[19] Keek, L., Iwakiri, W., Serino, M., Ballantyne, D. R., in’t Zand, J. J. M., and Strohmayer, T. E., "X-Ray Reflection and an Exceptionally Long Thermonuclear Helium Burst from IGR J17062-6143," ApJ 836, 111 (Feb. 2017).

[20] Keek, L. et al., "NICER Observes the Effects of an X-Ray Burst on the Accretion Environment in Aql X-1," ApJ 855, L4 (Mar. 2018).

[21] Uttley, P., Cackett, E. M., Fabian, A. C., Kara, E., and Wilkins, D. R., "X-ray reverberation around accreting black holes," A\&A Rev. 22, 72 (Aug. 2014).

[22] Zoghbi, A., Fabian, A. C., Reynolds, C. S., and Cackett, E. M., "Relativistic iron K X-ray reverberation in NGC 4151," MNRAS 422, 129-134 (May 2012).

[23] Kara, E., Alston, W. N., Fabian, A. C., Cackett, E. M., Uttley, P., Reynolds, C. S., and Zoghbi, A., "A global look at X-ray time lags in Seyfert galaxies," MNRAS 462, 511-531 (Oct. 2016).

[24] Kara, E. et al., "The corona contracts in a black-hole transient," Nature 565, 198-201 (Jan. 2019).

[25] Hoormann, J. K., Beheshtipour, B., and Krawczynski, H., "Testing general relativity's no-hair theorem with x-ray observations of black holes," Phys. Rev. D 93, 044020 (Feb. 2016). 
[26] Jiang, J., Bambi, C., and Steiner, J. F., "Testing the Kerr nature of black hole candidates using iron line reverberation mapping in the Cardoso-Pani-Rico framework," Phys. Rev. D 93, 123008 (June 2016).

[27] Miyamoto, S. and Kitamoto, S., "A jet model for a very high state of GX 339 - 4," ApJ 374, 741-743 (June 1991).

[28] Takizawa, M. et al., "Spectral and Temporal Variability in the X-Ray Flux of GS 1124-683, Nova Muscae 1991," ApJ 489, 272-283 (Nov. 1997).

[29] van der Klis, M., "Comparing Black Hole and Neutron Star Variability," Ap\&SS 300, 149-157 (Nov. 2005).

[30] van der Klis, M., [Rapid X-ray Variability], 39-112 (Apr. 2006).

[31] Morgan, E. H., Remillard, R. A., and Greiner, J., "RXTE Observations of QPOs in the Black Hole Candidate GRS 1915+105," ApJ 482, 993-1010 (June 1997).

[32] van der Klis, M., "Millisecond Oscillations in X-ray Binaries," ARA\&A 38, 717-760 (2000).

[33] Chakrabarti, S. K. and Molteni, D., "Smoothed Particle Hydrodynamics Confronts Theory: Formation of Standing Shocks in Accretion Disks and Winds around Black Holes," ApJ 417, 671 (Nov. 1993).

[34] Stella, L. and Vietri, M., "Lense-Thirring Precession and Quasi-periodic Oscillations in Low-Mass X-Ray Binaries," ApJ 492, L59-L62 (Jan. 1998).

[35] Stella, L., Vietri, M., and Morsink, S. M., "Correlations in the Quasi-periodic Oscillation Frequencies of Low-Mass X-Ray Binaries and the Relativistic Precession Model," ApJ 524, L63-L66 (Oct. 1999).

[36] Tagger, M. and Pellat, R., "An accretion-ejection instability in magnetized disks," A\&A 349, 1003-1016 (Sept. 1999).

[37] Wagoner, R. V., Silbergleit, A. S., and Ortega-Rodríguez, M., "'Stable" Quasi-periodic Oscillations and Black Hole Properties from Diskoseismology," ApJ 559, L25-L28 (Sept. 2001).

[38] Schnittman, J. D., Homan, J., and Miller, J. M., "A Precessing Ring Model for Low-Frequency Quasiperiodic Oscillations," ApJ 642, 420-426 (May 2006).

[39] Ingram, A., Done, C., and Fragile, P. C., "Low-frequency quasi-periodic oscillations spectra and LenseThirring precession," MNRAS 397, L101-L105 (July 2009).

[40] Cabanac, C., Henri, G., Petrucci, P.-O., Malzac, J., Ferreira, J., and Belloni, T. M., "Variability of X-ray binaries from an oscillating hot corona," MNRAS 404, 738-748 (May 2010).

[41] Lai, D. et al., "High-Frequency QPOs and Overstable Oscillations of Black-Hole Accretion Disks," in [Feeding Compact Objects: Accretion on All Scales], IAU Symposium 290, 57-61 (Feb. 2013).

[42] Belloni, T. M. and Stella, L., "Fast Variability from Black-Hole Binaries," Space Sci. Rev. 183, 43-60 (Sept. 2014).

[43] van der Klis, M., "Overview of QPOs in neutron-star low-mass X-ray binaries," Advances in Space Research 38, 2675-2679 (Jan. 2006).

[44] Ingram, A., van der Klis, M., Middleton, M., Done, C., Altamirano, D., Heil, L., Uttley, P., and Axelsson, M., "A quasi-periodic modulation of the iron line centroid energy in the black hole binary H1743-322," MNRAS 461, 1967-1980 (Sept. 2016).

[45] Ingram, A., van der Klis, M., Middleton, M., Altamirano, D., and Uttley, P., "Tomographic reflection modelling of quasi-periodic oscillations in the black hole binary H 1743-322," MNRAS 464, 2979-2991 (Jan. 2017). 
[46] et al., S. M., "Black hole spin measurements through the relativistic precession model: Xte j1550-564," Monthly Notices of the Royal Astronomical Society (2014).

[47] Miller, M. C., Lamb, F. K., and Psaltis, D., "Sonic-Point Model of Kilohertz Quasi-periodic Brightness Oscillations in Low-Mass X-Ray Binaries," ApJ 508, 791-830 (Dec. 1998).

[48] van Doesburgh, M., van der Klis, M., and Morsink, S. M., "The highest frequency kHz QPOs in neutron star low-mass X-ray binaries," MNRAS 479, 426-434 (Sep 2018).

[49] Barret, D. et al., "The Athena X-ray Integral Field Unit (X-IFU)," in [Space Telescopes and Instrumentation 2016: Ultraviolet to Gamma Ray], Proc. SPIE 9905, 99052F (July 2016).

[50] Pounds, K. A., Reeves, J. N., King, A. R., Page, K. L., O'Brien, P. T., and Turner, M. J. L., "A high-velocity ionized outflow and XUV photosphere in the narrow emission line quasar PG1211+143," MNRAS 345, 705-713 (Nov 2003).

[51] Tombesi, F., Cappi, M., Reeves, J. N., Palumbo, G. G. C., Yaqoob, T., Braito, V., and Dadina, M., "Evidence for ultra-fast outflows in radio-quiet AGNs. I. Detection and statistical incidence of Fe K-shell absorption lines," A\&A 521, A57 (Oct 2010).

[52] Nicastro, F., Kaastra, J., Krongold, Y., Borgani, S., Branchini, E., Cen, R., Dadina, M., Danforth, C. W., Elvis, M., Fiore, F., Gupta, A., Mathur, S., Mayya, D., Paerels, F., Piro, L., Rosa-Gonzalez, D., Schaye, J., Shull, J. M., Torres-Zafra, J., Wijers, N., and Zappacosta, L., "Observations of the missing baryons in the warm-hot intergalactic medium," Nature 558, 406-409 (Jun 2018).

[53] Planck Collaboration et al., "Planck 2018 results. VI. Cosmological parameters," arXiv e-prints , arXiv:1807.06209 (Jul 2018).

[54] Davé, R., Cen, R., Ostriker, J. P., Bryan, G. L., Hernquist, L., Katz, N., Weinberg, D. H., Norman, M. L., and O'Shea, B., "Baryons in the Warm-Hot Intergalactic Medium," ApJ 552, 473-483 (May 2001).

[55] Tilton, E. M., Danforth, C. W., Shull, J. M., and Ross, T. L., "The Low-redshift Intergalactic Medium as Seen in Archival Legacy HST/STIS and FUSE Data," ApJ 759, 112 (Nov 2012).

[56] Shull, J. M., Smith, B. D., and Danforth, C. W., "The Baryon Census in a Multiphase Intergalactic Medium: 30\% of the Baryons May Still be Missing," ApJ 759, 23 (Nov 2012).

[57] Lehner, N., Savage, B. D., Richter, P., Sembach, K. R., Tripp, T. M., and Wakker, B. P., "Physical Properties, Baryon Content, and Evolution of the Ly $\alpha$ Forest: New Insights from High-Resolution Observations at z \&lt; 0.4," ApJ 658, 680-709 (Apr 2007).

[58] Danforth, C. W., Stocke, J. T., and Shull, J. M., "Broad H I Absorbers as Metallicity-independent Tracers of the Warm-Hot Intergalactic Medium," ApJ 710, 613-633 (Feb 2010).

[59] Danforth, C. W. and Shull, J. M., "The Low-z Intergalactic Medium. III. H I and Metal Absorbers at z \&lt; 0.4," ApJ 679, 194-219 (May 2008).

[60] Narayanan, A., Savage, B. D., Wakker, B. P., Danforth, C. W., Yao, Y., Keeney, B. A., Shull, J. M., Sembach, K. R., Froning, C. S., and Green, J. C., "Cosmic Origins Spectrograph Detection of Ne VIII Tracing Warm-Hot Gas Toward PKS 0405-123," ApJ 730, 15 (Mar 2011).

[61] Nicastro, F., Mathur, S., and Elvis, M., "Missing Baryons and the Warm-Hot Intergalactic Medium," Science 319, 55 (Jan 2008).

[62] Fang, T., Buote, D. A., Humphrey, P. J., Canizares, C. R., Zappacosta, L., Maiolino, R., Tagliaferri, G., and Gastaldello, F., "Confirmation of X-ray Absorption by Warm-Hot Intergalactic Medium in the Sculptor Wall," ApJ 714, 1715-1724 (May 2010). 
[63] Zappacosta, L., Nicastro, F., Maiolino, R., Tagliaferri, G., Buote, D. A., Fang, T., Humphrey, P. J., and Gastaldello, F., "Studying the WHIM Content of Large-scale Structures Along the Line of Sight to H 2356-309," ApJ 717, 74-84 (Jul 2010).

[64] Fang, T., Marshall, H. L., Lee, J. C., Davis, D. S., and Canizares, C. R., "Chandra Detection of O VIII Ly $\alpha$ Absorption from an Overdense Region in the Intergalactic Medium," ApJ 572, L127-L130 (Jun 2002).

[65] Fang, T., Canizares, C. R., and Yao, Y., "Confirming the Detection of an Intergalactic X-Ray Absorber toward PKS 2155-304," ApJ 670, 992-999 (Dec 2007).

[66] Kovács, O. E., Bogdán, Á., Smith, R. a. K., Kraft, R. P., and Forman, W. R., "Detection of the Missing Baryons toward the Sightline of H1821+643," ApJ 872, 83 (Feb 2019).

[67] Steiner, A. W., Lattimer, J. M., and Brown, E. F., "The Neutron Star Mass-Radius Relation and the Equation of State of Dense Matter," ApJ 765, L5 (Mar. 2013).

[68] Ho, W. C. G., Kaplan, D. L., Chang, P., van Adelsberg, M., and Potekhin, A. Y., "Magnetic hydrogen atmosphere models and the neutron star RX J1856.5-3754," MNRAS 375, 821-830 (Mar. 2007).

[69] Özel, F. and Psaltis, D., "Spectral Lines from Rotating Neutron Stars," ApJ 582, L31-L34 (Jan 2003).

[70] Bauböck, M., Psaltis, D., and Özel, F., "Narrow Atomic Features from Rapidly Spinning Neutron Stars," ApJ 766, 87 (Apr. 2013).

[71] Strohmayer, T. E. and Markwardt, C. B., "EXO 1745-248 is an $11 \mathrm{~Hz}$ Eclipsing Pulsar," The Astronomer's Telegram 2929 (Oct. 2010).

[72] Yoneda, H., Done, C., Paerels, F., Takahashi, T., and Watanabe, S., "Search for gravitational redshifted absorption lines in LMXB Serpens X-1," MNRAS 475, 2194-2203 (Apr. 2018).

[73] Cottam, J., Paerels, F., and Mendez, M., "Gravitationally redshifted absorption lines in the X-ray burst spectra of a neutron star," Nature 420, 51-54 (Nov. 2002).

[74] Galloway, D. K., Lin, J., Chakrabarty, D., and Hartman, J. M., "Discovery of a 552 Hz Burst Oscillation in the Low-Mass X-Ray Binary EXO 0748-676," ApJ 711, L148-L151 (Mar. 2010).

[75] Lin, J., Özel, F., Chakrabarty, D., and Psaltis, D., "The Incompatibility of Rapid Rotation with Narrow Photospheric X-ray Lines in EXO 0748-676," ApJ 723, 1053-1056 (Nov. 2010).

[76] Weinberg, N. N., Bildsten, L., and Schatz, H., "Exposing the Nuclear Burning Ashes of Radius Expansion Type I X-Ray Bursts," ApJ 639, 1018-1032 (Mar. 2006).

[77] Kajava, J. J. E., Nättilä, J., Poutanen, J., Cumming, A., Suleimanov, V., and Kuulkers, E., "Detection of burning ashes from thermonuclear X-ray bursts," MNRAS 464, L6-L10 (Jan. 2017).

[78] Li, Z., Suleimanov, V. F., Poutanen, J., Salmi, T., Falanga, M., Nättilä, J., and Xu, R., "Evidence for the Photoionization Absorption Edge in a Photospheric Radius Expansion X-Ray Burst from GRS 1747-312 in Terzan 6," ApJ 866, 53 (Oct 2018).

[79] Rutledge, R. E., Bildsten, L., Brown, E. F., Pavlov, G. G., and Zavlin, V. E., "Variable Thermal Emission from Aquila X-1 in Quiescence," ApJ 577, 346-358 (Sept. 2002).

[80] Wijnands, R., Degenaar, N., and Page, D., "Cooling of Accretion-Heated Neutron Stars," Journal of Astrophysics and Astronomy 38, 49 (Sept. 2017).

[81] Ford, E. C., "Lag of Low-Energy Photons in an X-Ray Burst Oscillation: Doppler Delays," ApJ 519, L73-L75 (July 1999).

[82] Heyl, J. S., "R-modes on rapidly rotating, relativistic stars - II. Blackbody emission," MNRAS 361, 504510 (Aug. 2005). 
[83] Psaltis, D., Ozel, F., and Chakrabarty, D., "Prospects for measuring neutron-star masses and radii with x-ray pulse profile modeling," The Astrophysical Journal 787(2), 136 (2014).

[84] Ozel, F., Psaltis, D., Arzoumanian, Z., Morsink, S., and Baubock, M., "Measuring neutron star radii via pulse profile modeling with nicer," The Astrophysical Journal 832(1), 92 (2016).

[85] Aharonian, F. et al., "Hitomi X-ray observation of the pulsar wind nebula G21.5-0.9," PASJ (Apr. 2018).

[86] Borghese, A., Rea, N., Coti Zelati, F., Tiengo, A., Turolla, R., and Zane, S., "Narrow phase-dependent features in X-ray dim isolated neutron stars: a new detection and upper limits," MNRAS 468, 2975-2983 (July 2017).

[87] Ibrahim, A. I., Safi-Harb, S., Swank, J. H., Parke, W., Zane, S., and Turolla, R., "Discovery of Cyclotron Resonance Features in the Soft Gamma Repeater SGR 1806-20," ApJ 574, L51-L55 (Jul 2002).

[88] Rea, N., Israel, G. L., and Stella, L., "First evidence of a cyclotron feature in an anomalous X-ray pulsar," Nuclear Physics B Proceedings Supplements 132, 554-559 (Jun 2004).

[89] Gavriil, F. P., Dib, R., and Kaspi, V. M., "Activity From Magnetar Candidate 4U 0142+61: Bursts and Emission Lines," in [40 Years of Pulsars: Millisecond Pulsars, Magnetars and More], Bassa, C., Wang, Z., Cumming, A., and Kaspi, V. M., eds., American Institute of Physics Conference Series 983, 234-238 (Feb 2008).

[90] Tiengo, A., Esposito, P., Mereghetti, S., Turolla, R., Nobili, L., Gastaldello, F., Götz, D., Israel, G. L., Rea, N., Stella, L., Zane, S., and Bignami, G. F., "A variable absorption feature in the X-ray spectrum of a magnetar," Nature 500, 312-314 (Aug 2013).

[91] Zane, S., Turolla, R., Stella, L., and Treves, A., "Proton Cyclotron Features in Thermal Spectra of Ultramagnetized Neutron Stars," ApJ 560, 384-389 (Oct 2001).

[92] Ho, W. C. G. and Lai, D., "Atmospheres and spectra of strongly magnetized neutron stars," MNRAS 327, 1081-1096 (Nov 2001).

[93] Lai, D. and Ho, W. C. G., "Resonant Conversion of Photon Modes Due to Vacuum Polarization in a Magnetized Plasma: Implications for X-Ray Emission from Magnetars," ApJ 566, 373-377 (Feb 2002).

[94] Özel, F., "The Effect of Vacuum Polarization and Proton Cyclotron Resonances on Photon Propagation in Strongly Magnetized Plasmas," ApJ 583, 402-409 (Jan 2003).

[95] Özel, F., Psaltis, D., Arzoumanian, Z., Morsink, S., and Bauböck, M., "Measuring Neutron Star Radii via Pulse Profile Modeling with NICER," ApJ 832, 92 (Nov 2016).

[96] Watts, A. L. et al., "Colloquium: Measuring the neutron star equation of state using x-ray timing," Reviews of Modern Physics 88, 021001 (Apr. 2016).

[97] Bogdanov, S., Lamb, F. K., Mahmoodifar, S., Miller, M. C., Morsink, S. M., Riley, T. E., Strohmayer, T. E., Tung, A. K., Watts, A. L., Dittmann, A. J., Chakrabarty, D., Guillot, S., Arzoumanian, Z., and Gendreau, K. C., "Constraining the Neutron Star Mass-Radius Relation and Dense Matter Equation of State with NICER. II. Emission from Hot Spots on a Rapidly Rotating Neutron Star," ApJ 887, L26 (Dec 2019).

[98] Miller, M. C., "The Case for PSR J1614-2230 as a NICER Target," ApJ 822, 27 (May 2016).

[99] Wolff, M., Guillot, S., Ho, W. C., and Ray, P. S., "NICER Observations of the Massive Millisecond Pulsar PSR J1614-2230," in [American Astronomical Society Meeting Abstracts \#233], American Astronomical Society Meeting Abstracts 233, 153.13 (Jan 2019).

[100] Gierliński, M., Done, C., and Barret, D., "Phase-resolved X-ray spectroscopy of the millisecond pulsar SAX J1808.4-3658," MNRAS 331, 141-153 (Mar 2002). 